\title{
The usefulness of pre-employment and pre-deployment psychological screening for disaster relief workers: a systematic review
}

Elena Opie*, Samantha Brooks, Neil Greenberg and G. James Rubin

\begin{abstract}
Background: Individuals who conduct disaster relief work overseas are exposed to a variety of traumatic events that can cause distress and trigger psychological illnesses. Identification of which disaster relief workers may be at risk of experiencing psychological distress or mental health disorders is frequently carried out through preemployment or pre-deployment psychological screening. The primary objective of our review was to assess the evidence for pre-employment and pre-deployment psychological screening of relief workers who work in disaster situations. We aimed to identify specific pre-employment and pre-deployment characteristics that predict impaired wellbeing of an individual following engaging in disaster-related work.

Methods: A combined list of search terms was composed relating to disaster-related occupations, screening methods, psychological disorders, and study design. The databases used were PsycINFO, MEDLINE, EMBASE, and GlobalHealth. We included studies that used cross-sectional or longitudinal study designs; were published in the English language in peer-reviewed academic journals; reported on the association between pre-employment and pre-deployment features and post-deployment psychological disorders or distress; considered any occupational groups responding to a specified, discrete crisis; and used at least one validated measure of distress or disorder. We extracted data on the author; year of publication; disaster description; country of study; study design; population sample; disorder(s) outcome and the measures used; and results.
\end{abstract}

Results: Sixty-two, high-quality studies were included in the review. Forty-one potential predictors were identified. Of these, only volunteer status and previous history of mental illness and life stressors emerged as reliable predictors of distress or disorder.

Conclusion: The results suggest that whilst it is attractive to screen for pre-employment and pre-deployment indicators of resilience, the evidence base for doing so is weak. At best, this sort of screening can only weakly suggest vulnerability and at worst may result in discrimination. Until better evidence about its usefulness becomes available, employers should exercise caution over its use.

Keywords: Resilience factors, Predictors, Disaster relief workers, Psychological distress, Psychological disorder

\footnotetext{
* Correspondence: elena.opie@gmail.com

NIHR Health Protection Research Unit in Emergency Preparedness and Response, King's College London, Weston Education Centre, Cutcombe Rd, London SE5 9RJ, UK
}

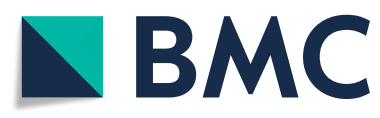

(c) The Author(s). 2020 Open Access This article is licensed under a Creative Commons Attribution 4.0 International License, which permits use, sharing, adaptation, distribution and reproduction in any medium or format, as long as you give appropriate credit to the original author(s) and the source, provide a link to the Creative Commons licence, and indicate if changes were made. The images or other third party material in this article are included in the article's Creative Commons licence, unless indicated otherwise in a credit line to the material. If material is not included in the article's Creative Commons licence and your intended use is not permitted by statutory regulation or exceeds the permitted use, you will need to obtain permission directly from the copyright holder. To view a copy of this licence, visit http://creativecommons.org/licenses/by/4.0/. The Creative Commons Public Domain Dedication waiver (http://creativecommons.org/publicdomain/zero/1.0/) applies to the data made available in this article, unless otherwise stated in a credit line to the data. 


\section{Background}

Individuals who conduct disaster relief work overseas may be exposed to traumatic events that can cause distress and trigger psychological illnesses such as posttraumatic stress disorder (PTSD), depression and anxiety disorders [1-6]. Disaster relief workers include professionals such as firefighters, police, search-and-rescue and relief teams, and medical personnel alongside volunteer relief workers with no formal training. Previous research has identified that the rates of PTSD amongst these populations range from 8 to 25\% [7-9]. Whilst these figures underline the risk associated with relief work, they also indicate that only a minority of traumaexposed workers develop psychological distress and illness [10]. This raises the question of whether it is possible to predict which individuals will be susceptible to psychological distress or disorder following the trauma exposure that can occur in disaster relief work.

Identification of disaster workers who might be vulnerable to developing post-trauma mental health disorders is often attempted via pre-employment or predeployment psychological screening methods. Those identified as being vulnerable may be prevented from taking up a disaster worker role, be more closely monitored during their work or be restricted in the duties they can undertake. However, pre-deployment psychological screening has a chequered history. In World War II, the USA identified around 2 million men as being at risk of breaking down under the stress of combat, the majority of whom subsequently proved to be resilient, effective soldiers [11]. Recent efforts to screen military personnel have demonstrated a similar lack of precision [12]; a study [13] screened military personnel for mental disorders pre-deployment, a frequently reported riskfactor [14], only to find that it had low positive predictive value for developing mental health disorders postdeployment.

The apparent lack of effectiveness of screening tools has encouraged researchers to expand their search for risk and resilience factors in populations working in trauma prone environments. A recent review on screening within the emergency services [15] identified multiple predictors of mental health including neuroticism, pre-existing psychopathology, trauma history, maladaptive coping styles including catastrophic thinking and rumination, and social factors, such as substance related disorders. Still, the study came to conclude that there were no reliable ways to enhance personnel selection through screening and the authors deduced that further research on this topic was needed. The review [15], however, was limited to individuals working in general emergency service roles rather than disaster responders with most of the identified studies only including trainee police officers and firefighters.
The primary objective of our review is to assess the evidence for pre-employment and pre-deployment psychological screening of disaster response workers. We aimed to identify specific characteristics, which can be detected pre-engagement, that predict impaired wellbeing of an individual following their work.

\section{Methods}

This systematic review was conducted with reference to the PRISMA criteria [16].

\section{Protocol and registration}

An unpublished protocol was developed by the research team and was reviewed and edited by each member. The protocol was not registered.

\section{Study selection}

We included studies if they met all the following criteria:

- Published in peer-reviewed, academic journals;

- Published in English;

- Considered any occupational group responding to a specified, discrete crisis;

- Reported on the association between preemployment and/or pre-deployment features and post-deployment psychological disorders or distress;

- Primary, quantitative research-based longitudinal or cross-sectional study designs;

- Used at least one validated measure of distress or disorder.

Only published papers written in the English language were included in the review due to the resources available to conduct this systematic review. For the same reason, grey literature was not included, also to ensure that all studies included had gone through a peer review process. There were no limits on the year of publication or of follow-up time to include all the relevant research. Studies relating to military personnel were included if the personnel were studied in relation to a discrete humanitarian incident or disaster, but not if they were studied in relation to a combat or peacekeeping missions, since the nature of these duties would provide data that was not relevant to the research question.

\section{Conducting the review}

A combined list of search terms was composed by examining relevant past literature (Additional file 1). The terms related to disaster-related occupations, screening methods, psychological disorders, and study design. In August 2018, one author (EO) conducted the search using the following databases: PsycINFO (1806-2018), MEDLINE (1946-2018), EMBASE (1980-2018), GlobalHealth (1966-2018). The thesaurus of each database was 
checked regarding each term to ensure applicability for that database. A final search was conducted by EO on the 18/10/2018 to update the review. EO also undertook thorough reference checking and tracking, including forward citation tracking, of each included study and of relevant systematic reviews. In turn, this strengthened the search and greatly reduced the risk of missing relevant papers. All resulting citations were downloaded to EndNote@ software version X7 [17].

Bibliographic searching was completed in August 2018 and a total of 8426 potentially relevant papers were identified (including the papers identified via reference checking and tracking). Upon removing all duplicate studies, 5627 studies remained. All titles and abstracts were screened by EO, and the papers excluded if they did not correspond to the inclusion criteria; 227 studies remained. After reviewing the full papers, 165 papers were excluded and a total of 62 papers were included in the review. Please see Additional file 3 for the Flow Diagram of the study selection process.

The general causes of exclusion were that many of the studies were not of longitudinal or cross-sectional design, did not include completion of pre-deployment measures, were based on military samples working in combat or peacekeeping missions, were not based on a specified, discrete crisis and/or did not use at least one validated measure.

\section{Data extraction, quality appraisal, and data synthesis}

EO was trained on how to use the data collection form by SKB [18]. EO piloted using the form with one paper, which SKB then reviewed; discussion for any areas for improvement was had. Coding instructions were clearly stated on the data collection form. Using the data collection form, all papers underwent data extraction by EO (Additional File 4. Main Results Table). A small number of these papers had data independently extracted by SKB. Upon a comparison of the extracted data by both researchers, consensus was generally high. Any disagreements were solved by discussion between the research team.

We extracted and recorded the following variables from each included study: author, year of publication; disaster description; country of study; study design; time assessed since deployment/follow-up time; population sample (' $n$ ' and demographic data including gender split, age range, mean age, job role); disorder outcome(s) and the measures used; predictive factors including key results.

We assessed the quality of each study in three areas: study design; data collection and methodology; and results. Our quality assessment tool (Additional file 2) was designed for a previous review [18] and was informed by existing quality appraisal tools $[19,20]$. Each study was given an overall score as a percentage, based on the number of 'yes' responses, with a higher score indicating better quality.

For data synthesis, thematic analysis was used to group predictive factors into a typology. Topics we accepted as "themes" were required to be identified by at least two studies to be discussed in the in-text results; predictor variables explored by only a single study are reported only in the tables.

Thematic analysis was also used to group psychiatric distresses into a typology; in Tables 1-7, secondary trauma was re-grouped into PTSD given that the symptoms are very similar [81] and acute stress was regrouped into stress. It should be noted that studies often reported psychological distress or morbidity as the outcome without stating whether participants were likely to have a specified mental health diagnosis or not.

\section{Results}

\section{Study characteristics}

The initial search yielded 8386 studies. Thorough reference checking and tracking, including forward citation tracking of each included study and relevant systematic reviews, identified another 40 citations. Sixty-two studies met our inclusion criteria.

The details of each study and their findings are given in Table 1 . The majority $(n=60)$ were cross-sectional; just two were prospective. Most $(n=41)$ focussed on large-scale disasters, natural or accidental (e.g. earthquakes, air crashes), 19 studies focussed on terrorism and 14 examined the 11th September attacks in the USA. The included studies used a variety of measures to assess the mental health of all participants, for a brief description of each measure, please see the Main Results Table, Additional file 4.

Overall, 41 different potential predictors were identified. We categorised these as relating to demographics; history of mental illness; history of trauma; previous experience as a disaster relief worker; personality traits; coping strategies; other. The proportion of studies supporting or opposing that a predictor is associated with mental-ill health is reported. Where only one study examined a predictor, it has not been reported in text. Please see the corresponding tables for this data.

\section{Risk of bias}

Most included studies in the review obtained high quality scores (mean: $84.5 \%$; range 68.8 to $100 \%$ ). Please see the Main Results Table (Additional file 4) for the quality obtained by each study. The most common weaknesses were, not stating the inclusion or exclusion criteria, not describing reasons for loss to follow-up and, not reporting appropriate caveats in the interpretation of results. Given the high quality of the research in this area, it was 
Table 1 Demographics as a predictor of psychological distress following disaster relief work

\begin{tabular}{|c|c|}
\hline Disorder & Papers reporting significant association with disorder / papers reporting non-significant association with disorder \\
\hline PTSD & $\begin{array}{l}\text { Age }[7,21-35] /[8,36-54] \\
\text { Gender }[23,25,28-30,35,37,43,53-55] /[8,21,22,31,33,36,38-42,46,48-50,52,56,57] \\
\text { Ethnicity }[22,25,28-30] /[23,31,36,44,49,51,54,57] \\
\text { Education }[22,25,28-31] /[8,33,34,39,40,42,44,49,51-54,58] \\
\text { Marital status }[22,24,27-30,42,51] /[7,8,26,33,34,36,38-40,43,44,47,53,57] \\
\text { Income }[28,30] /[23,29,33,49] \\
\text { Social class / }[32] \\
\text { Religious group / }[54]\end{array}$ \\
\hline Anxiety & $\begin{array}{l}\text { Age }[48,54] /[21,22,47,51] \\
\text { Gender }[22,54] /[21,48] \\
\text { Ethnicity }[22] /[51,54] \\
\text { Education }[22] /[51,54] \\
\text { Marital status }[22] /[47,51] \\
\text { Religious group / [54] }\end{array}$ \\
\hline Depression & $\begin{array}{l}\text { Age }[22,54,59] /[21,39,42,43,47,48,50-52] \\
\text { Gender }[22,48,54] /[21,39,42,43,50,52,59] \\
\text { Ethnicity }[22,59] /[51,54] \\
\text { Education }[22,52] /[39,42,51,54,59] \\
\text { Marital status }[22,42,47] /[39,43,51,59] \\
\text { Religious group / [54] }\end{array}$ \\
\hline Psychological distress / morbidity & $\begin{array}{l}\text { Age }[7,26,41,60] /[23,24,49,61-63] \\
\text { Gender }[23] /[41,49,55,61,63] \\
\text { Ethnicity / }[23,49,63] \\
\text { Education / }[49,58,63] \\
\text { Marital status }[7] /[24,26,61] \\
\text { Income / }[23,49] \\
\text { Social class / [62] }\end{array}$ \\
\hline Somatic symptoms & $\begin{array}{l}\text { Age / [21, 47, 51, 54] } \\
\text { Gender [54] / [21] } \\
\text { Ethnicity [51] / [54] } \\
\text { Education / [51,54] } \\
\text { Marital status }[47] /[51,54] \\
\text { Religious group / [54] }\end{array}$ \\
\hline Burnout & $\begin{array}{l}\text { Age }[21] /[36,49,54] \\
\text { Gender }[21,54] /[36,49,55,57] \\
\text { Ethnicity / [36, 49, 54,57] } \\
\text { Education / [49,54] } \\
\text { Marital status }[36,57] \\
\text { Income / [49] } \\
\text { Religious group / [54] }\end{array}$ \\
\hline Stress & $\begin{array}{l}\text { Age }[42] /[46,59] \\
\text { Gender / [42, 46, 59] } \\
\text { Ethnicity / [59] } \\
\text { Education / [42, 59] } \\
\text { Marital status }[42] /[59]\end{array}$ \\
\hline Peritraumatic dissociation & $\begin{array}{l}\text { Age }[64] /[38,39,59] \\
\text { Gender / [38, 39, 56, 59] } \\
\text { Ethnicity / [59] } \\
\text { Education / [39, 59] } \\
\text { Marital status / [38, 39, 59] }\end{array}$ \\
\hline Fatigue & $\begin{array}{l}\text { Age [63] } \\
\text { Gender [63] } \\
\text { Ethnicity / [63] } \\
\text { Education / [63] }\end{array}$ \\
\hline Quality of life & $\begin{array}{l}\text { Age / [63] } \\
\text { Gender / [63] } \\
\text { Ethnicity / [63] } \\
\text { Education / [63] }\end{array}$ \\
\hline Hostility & $\begin{array}{l}\text { Age / [51, 52] } \\
\text { Gender / [52] } \\
\text { Ethnicity / [51] } \\
\text { Education / [51, 52] } \\
\text { Marital status / [51] }\end{array}$ \\
\hline
\end{tabular}


Table 1 Demographics as a predictor of psychological distress following disaster relief work (Continued)

\begin{tabular}{ll}
\hline Disorder & Papers reporting significant association with disorder / papers reporting non-significant association with disorder \\
\hline Alcohol disorder & Age / [43] \\
& Gender / [43] \\
Marital status / [43] \\
Resilience & Age / [49] \\
& Gender / [49] \\
& Ethnicity / [49] \\
& Education / [49] \\
Income / [49]
\end{tabular}

not possible to weigh the findings of individual studies by quality in our narrative synthesis.

\section{Associations between demographics and distress and morbidity}

Table 1 shows the association between demographics and distress or disorder. There is mixed evidence for an association for age. Most findings revealed no association between age and PTSD (19 out of 34), anxiety (four out of six), depression (nine out of 12), psychological distress/morbidity (six out of 10), somatic symptoms (four out of four), burnout (three out of four, with the latter being a lower quality study [21]), stress (two out of three), peritraumatic dissociation (three out of four) or hostility (two out of two). Still, some studies did identify that being older was associated with stronger severity and higher probability of PTSD (seven out of 34) alongside a worse trajectory of this disorder (two out of 34) and, higher probability of general psychological distress/morbidity (three out of 10). Other studies identified that youth was associated with a higher probability of PTSD (six out of 34), anxiety (two out of six) and depression (two out of 12).

There is mixed evidence for an association for gender. The majority revealed no association between gender and PTSD (17 out of 28), depression (seven out of 10), psychological distress/morbidity (five out of six), peritraumatic dissociation (four out of four), burnout (four out of six, with one of the two contradictory papers being a lower quality paper [21]) and, stress (three out of three). A minority of studies found that being a woman was associated with a higher probability of depression (two out of 10), PTSD (eight out of 28) with a worse trajectory (two out of 28) and, higher severity of burnout (two out of six). Mixed findings were found regarding anxiety and gender; two studies out of four found that being a woman was associated with a higher probability of this condition whilst two others found no association. It is worth noting that for the latter, one of these two studies [21] was one of the few poorer quality studies.

There is mixed evidence for an association for ethnicity. Most findings revealed no association between ethnicity and PTSD (eight out of 13), anxiety (two out of three), general psychological distress or morbidity (three out of three) or burnout (four out of four). However, a minority of papers found that non-White participants were more likely to report PTSD (three out of 13) with a worse trajectory $[29,30]$. Mixed findings were found regarding depression and ethnicity; two studies out of four found that non-White participants were associated with a higher probability of this condition whilst two others found no association.

There is little evidence for an association for education. Most findings revealed no association between the level of education and PTSD (13 out of 19), anxiety (two out of three), depression (five out of seven), psychological distress/morbidity (three out of three), somatic symptoms (two out of two), stress (two out of two), peritraumatic dissociation (two out of two), burnout (two out of two), or hostility (two out of two). Still, some studies did find that having less education was associated with a higher probability of PTSD (four out of 19).

There is limited evidence for an association for marital status. Most findings revealed no association between marital status and PTSD (14 out of 22), anxiety (two out of three), depression (four out of seven), psychological distress or morbidity (three out of four), somatic symptoms (two out of three), or peritraumatic dissociation (three out of three). Still, there was some limited evidence that being unmarried, or not cohabiting, was associated with a higher probability of burnout (two out of two) and a higher probability and severity of depression (three out of seven) and PTSD (three out of three) with a worse trajectory [22, 28-30].

There is little evidence for associations between income, social class, or religious affiliation and psychiatric distress or morbidity. The majority of studies found that income was not associated with PTSD (four out of six) nor was it associated with psychological distress, (two out of two).

\section{Associations between history of mental illness and distress and morbidity}

Table 2 shows there to be strong evidence for an association between history of mental illness and distress or disorder. A history of mental illness was associated with higher severity and probability of PTSD (10 out of 11) with a worse trajectory $[29,30,45]$, anxiety and depression (for both two out of three). 
Table 2 History of mental illness as a predictor of psychological distress following disaster relief work

\begin{tabular}{ll}
\hline Disorder & Papers reporting significant association with disorder / papers reporting non-significant association with disorder \\
\hline PTSD & {$[27,29,30,32,34,35,45,65-67] /[68]$} \\
Anxiety & {$[65,67] /[68]$} \\
Depression & {$[65,67] /[68]$} \\
Psychological distress / morbidity & {$[65]$} \\
Alcohol and drug disorder & {$[67]$} \\
\hline
\end{tabular}

\section{Associations between history of trauma exposure and distress and morbidity}

Table 3 shows there to be evidence for an association between history of trauma exposure and distress or disorder. Studies found that history of trauma was marginally more associated with higher severity and probability of PTSD (nine out of 17), anxiety (two out of three), and depression (three out of five); the evidence was mixed for burnout (two our of four). A history of trauma that was similar to the trauma experienced during the disaster relief work was not associated with PTSD (three out of three). There is strong evidence that a history of life stressors is a predictor for psychiatric distress or disorder post-deployment. Studies described life stressors as, for example, "death of, or separation from, close relatives, major systemic disease or problems at work" [28, 60]. History of life stressors was associated with higher probability of PTSD (nine out 17) and psychological distress and morbidity (three out of three).

\section{Associations between previous experience with disaster relief work and distress and morbidity}

Table 4 shows there to be mixed evidence for an association between previous experience with disaster relief work and psychiatric distress or disorder. The majority of studies found that less experience was associated with higher post-trauma rates of PTSD (nine out of 11), psychological distress (two out of six) and peritraumatic dissociation (two out of four). Other studies found that more experience was associated with higher rates of PTSD (three out of 11) and general psychological distress and morbidity (four out of six). Other studies found no association between experience and PTSD (eight out of 19), anxiety (five out of five), depression (eight out of eight) or psychological distress or morbidity (two out of two).

\section{Associations between personality traits and distress and morbidity}

Table 5 shows there to be mixed evidence for an association between personality traits and psychiatric distress or disorder. Hardiness, defined as believing one is in control of one's life, that commitment to goals results in positive outcomes, and that daily stressors should be viewed as challenges [76], was associated with lower levels of psychological distress (two out of two). Neuroticism was associated with a higher probability of PTSD (three out of four with the fourth [68] being of lower quality rating), and severity of psychological distress (two out of two). Two studies, one [68] of lower quality rating, found that extraversion was not associated with PTSD (two out of three), but that adjustment, defined as

Table 3 History of trauma as a predictor of psychological distress following disaster relief work

\begin{tabular}{ll}
\hline Disorder & Papers reporting significant association with disorder / papers reporting non-significant association with disorder \\
\hline PTSD & {$[27,29,35,47,49,54,62,65,66] /[33,36,37,40,45,46,50,57]$} \\
& Similar / $[45,62,69]$ \\
& Dissimilar $[69]$ \\
& History of life stressors $[28,30,32,36,45] /[29]$ \\
& {$[47,54] /[65]$} \\
Anxiety & {$[47,54,65] /[50,59]$} \\
Depression & {$[62] /[49,65]$} \\
Psychological distress / morbidity & Similar / [69] \\
& Dissimilar [69] \\
& History of life stressors [60-62] \\
Stress & {$[59] /[46]$} \\
Somatic symptoms & {$[47] /[54]$} \\
Burnout & {$[36,54] /[49,57]$} \\
Peritraumatic dissociation & History of life stressors [36] \\
Resilience & /59] \\
\hline
\end{tabular}


Table 4 Previous experience as a disaster relief worker as a predictor of psychological distress following disaster relief work

\begin{tabular}{ll}
\hline Disorder & Papers reporting significant association with disorder / papers reporting non-significant association with disorder \\
\hline PTSD & {$[7,26,34,37-39,42,70-73] /[29,39,48,50,51,65,68,74]$} \\
& Success in prior disaster relief work / $[65]$ \\
Anxiety & $/[48,51,65,68,74]$ \\
& Success in prior disaster relief work / [65] \\
& $/[39,42,48,50,51,65,68,74]$ \\
Depression & Success in prior disaster relief work $[65]$ \\
Psychological distress / morbidity & {$[7,26,60,61,73,75] /[65,70]$} \\
& Success in prior disaster relief work / $[65]$ \\
Stress & $/[42]$ \\
Peritraumatic dissociation & {$[38,64] /[38,39]$} \\
Somatic symptoms & $/[51]$ \\
Hostility & $/[51]$
\end{tabular}

emotional stability [73], was associated with PTSD, though not with psychological distress/morbidity (two out of two).

\section{Associations between coping mechanisms and distress and morbidity}

Table 6 shows the association between coping mechanisms with disaster relief work and distress or disorder. Fifteen different components of coping were tested by only two studies, with all findings revealing no or conflicting evidence.

\section{Associations between other factors and distress and morbidity}

Table 7 shows there to be mixed evidence for an association between other factors and distress or disorder. Having children was not associated with burnout (two out of two). Having an external locus of control, defined as believing outside forces are in control, was associated with higher rates of PTSD (two out of two) and psychological distress (three out of three). Voluntary status when engaging with disaster relief work was associated with higher PTSD rates (three out of four), but not psychological distress (two out of three) and, professional status was not associated with PTSD (three out

Table 5 Personality traits as predictors of psychological distress following disaster relief work

\begin{tabular}{|c|c|}
\hline Disorder & Papers reporting significant association with disorder / papers reporting non-significant association with disorder \\
\hline 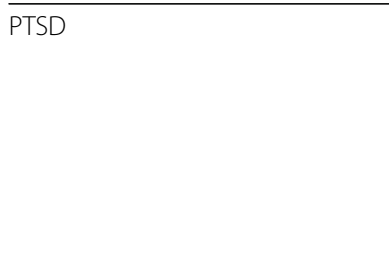 & $\begin{array}{l}\text { Hardiness [76] } \\
\text { Tough mindedness / [68] } \\
\text { Extraversion }[44] /[45,68] \\
\text { Neuroticism }[32,44,45] /[68] \\
\text { Dissimulation / [68] } \\
\text { Adjustment }[73,75] \\
\text { Avoidance }[45] \\
\text { Psychoticism [44] }\end{array}$ \\
\hline Anxiety & $\begin{array}{l}\text { Tough mindedness / [68] } \\
\text { Extraversion / [68] } \\
\text { Neuroticism [68] } \\
\text { Dissimulation / [68] }\end{array}$ \\
\hline Depression & $\begin{array}{l}\text { Tough mindedness / [68] } \\
\text { Extraversion / [68] } \\
\text { Neuroticism [68] } \\
\text { Dissimulation / [68] }\end{array}$ \\
\hline Psychological distress / morbidity & $\begin{array}{l}\text { Hardiness }[61,76] \\
\text { Conscientiousness [60] } \\
\text { Neuroticism }[60,77] \\
\text { Extroversion }[60] \\
\text { Adjustment }[73,75]\end{array}$ \\
\hline Peritraumatic dissociation & $\begin{array}{l}\text { Adjustment [64] } \\
\text { Prudence [64] } \\
\text { Ambition [64] } \\
\text { Identity [64] } \\
\text { Adaptive [64] }\end{array}$ \\
\hline
\end{tabular}


Table 6 Coping strategies as a predictor of psychological distress following disaster relief work

\begin{tabular}{ll}
\hline Disorder & Papers reporting significant association with disorder / papers reporting non-significant association with disorder \\
\hline PTSD & Confrontive coping / [7, 26] \\
& Seeking social support / [7, 26] \\
& Self-control / [7, 26] \\
& Accepting responsibility / [7, 26] \\
& Avoidance $[7] /[14]$ \\
& Planned problem solving / [7, 26] \\
& Positive reappraisal $[7] /[26]$ \\
& Confrontive coping $[7,26]$ \\
& Seeking social support $[14] /[7]$ \\
& Self-control / [7, 26] \\
Psychological distress / morbidity & Accepting responsibility [26] / [7] \\
& Avoidance $[26] /[7]$ \\
& Planned problem solving [26] / [7] \\
& Positive reappraisal $[26] /[7]$ \\
&
\end{tabular}

of four) anxiety (two out of two), or psychological distress (three out of four).

\section{Discussion}

To the best of our knowledge, this is the first systematic review investigating the potential usefulness of pre-employment and pre-deployment screening for predictors of psychological distress or disorder amongst disaster relief staff. We were able to identify and combine the findings from 62 studies. In general, demographics, previous experience as a disaster relief worker, personality traits, coping strategies, history of trauma, and having children have little evidence to support them as predictors of psychological vulnerability. Conversely, voluntary status, previous history of mental illness and previous life stressors spanning from in the past 3 months to throughout life, did emerge as reliable predictors. Locus of control, attachment style, childhood environment, and whether a person is reassured by physicians when unwell and experiencing symptoms were also identified as possible predictors and might be worthy of additional research as relatively few studies have assessed these features.

Table 7 Other predictors of psychological distress following disaster relief work

\begin{tabular}{|c|c|}
\hline Disorder & Papers reporting significant association with disorder / papers reporting non-significant association with disorder \\
\hline PTSD & $\begin{array}{l}\text { Having children }[54,78] /[36] \\
\text { Locus of control }[73,75] \\
\text { Voluntary status }[8,70,79] /[58] \\
\text { Professional status }[65] /[8,58,79] \\
\text { Attachment style }[49,55,78] \\
\text { Childhood environment }[53]\end{array}$ \\
\hline Anxiety & $\begin{array}{l}\text { Having children / [54] } \\
\text { Voluntary status }[79] \\
\text { Professional status / [65, 79] }\end{array}$ \\
\hline Depression & $\begin{array}{l}\text { Having children [54] / [59] } \\
\text { Professional status / [65] }\end{array}$ \\
\hline Psychological distress / morbidity & $\begin{array}{l}\text { Locus of control }[73,75,80] \\
\text { Professional status }[70] /[58,65,79] \\
\text { Voluntary status }[79] /[58,70] \\
\text { Reassured by physicians }[63] \\
\text { Attachment style }[77,78] /[49]\end{array}$ \\
\hline Stress & Having children / [59] \\
\hline Somatic symptoms & Having children / [54] \\
\hline Burnout & Having children / $[36,54]$ \\
\hline Peritraumatic dissociation & $\begin{array}{l}\text { Locus of control [64] } \\
\text { Having children / [59] }\end{array}$ \\
\hline Fatigue & Reassured by physicians [63] \\
\hline Quality of life & Reassured by physicians [63] \\
\hline Resilience & Attachment style [49] \\
\hline
\end{tabular}


Our findings that gender, ethnicity, education and marital status, were not associated with psychological vulnerability was surprising. These results deviate from previous findings. Both being a woman and belonging to an ethnic minority have often been identified as vulnerability factors for psychopathology as such individuals may be more susceptible to life-time stress [79, 82]. Similarly, our finding that those with lower education were not more at risk of psychological distress is contrary to other researcher's views that less educated people are more vulnerable to trauma because they have a reduced capacity to make sense of difficult experiences [83]. It was also unexpected to find that marital status was not associated with vulnerability; being married or in a long term relationship has been assumed to be associated with greater social support, which in turn is associated with reduced vulnerability to distress [84]. These findings suggest that such demographic variables are less relevant as predictors of distress or disorder among those people who choose to work in the field of disaster response, perhaps reflecting a generally high level of resilience among the group.

Our findings reveal clear evidence that individuals with a history of mental illness are more vulnerable to psychological distress following disaster relief work. Individuals who have a history of trauma and/or significant life stressors are also susceptible, although the evidence on this was less clear cut. These findings do not correspond with those of a systematic review exploring emergency service workers [15], which found that workers with preexisting mental illnesses or trauma were no more vulnerable than those without. This suggests that emergency services have procedures in place, such as mental health training for managers [85], to protect employees with such predisposing factors. It is likely that such procedures are not present for disaster relief workers.

Alternatively, the divergent findings might be explained by differences in the recruitment procedures for the two roles, resulting in qualitatively different populations, differences in training, or differences in the type of stressors the two groups are exposed to. Practical difficulties exist in basing pre-deployment screening on these findings however. First, those studies that explored life stressors used a range of time-periods in their measures, from life-stressors experienced in the past three to 6 months to those experienced at any point in the participant's life. Excluding or supporting all employees who have ever experienced a life stressor would be impractical and probably detrimental; it would prevent people who have experienced prior challenges from using their experience as a disaster worker. The evidence from this review suggests that people who have experienced significant life stressors should have their fitness to deploy carefully scrutinised and their mental health carefully monitored. Second, although a robust association was found between a history of mental illness and future mental health problems following humanitarian or disaster work, it remains unclear whether the increased risk of developing mental health problems post deployment is only related to recent episodes of ill mental health or not. Once again, we suggest that having a prior mental health history should not be seen as a bar to deployment, but as an indicator that someone's fitness to deploy should be carefully scrutinised by suitably experienced occupational health professionals and their mental health carefully monitored.

As suggested by Brooks and colleagues [86], volunteers in our review were found to be more prone to postdisaster psychopathology than those with a professional status. This may be linked to their lack of experience or training or that the organisations that make use of volunteer workers do not have well-established support mechanisms in place compared to those who employ professional disaster responders. Our results suggest that organisations should take particular care to prepare, support and monitor all disaster response staff, volunteer or professional. Methods for enabling this, such as organisational training and mental health education are discussed below.

Further research is needed before utilising many other potential predictors identified in this review for identifying pre-deployment vulnerability. These include parental status, attachment styles, childhood environment, external locus of control, and being reassured by physicians, all of which were found to increase vulnerability, although only in a small number of studies.

Few studies in this review investigated the impact of social support on psychological distress, however, the findings do suggest that it is an effective protective factor amongst relief workers $[7,26]$. This is supported by the literature; perceived and actual social support has the potential to alleviate psychological symptoms [8790]. Indeed, it is vital that relief work organisations create a culture of social support and openness about psychological difficulties amongst rescue workers [88, 89].

One approach is to encourage organisations to develop policy on mental health education for relief workers predeployment. Our results suggest that such education should focus on mental health literacy, the risk relief work poses, possible risk factors (e.g. prior life stressors and/or prior mental health problems), early warning signs, relaxation techniques and, available support services and their contact details; such education would enable recognition of ill mental health and swift actiontaking [88]. Organisations would be well advised to encourage staff to talk about mental health which may help empower them to seek support $[88,89]$. We would also suggest ensuing that program managers have access to 
mental-health training and well-developed protocols for differential responses based on the level of presenting problems and any identified risk to self or others [85]. As a result of these efforts, volunteers and professionals would feel well-prepared and supported and the culture of openness and mental health literacy further enforced [88].

Overall, our findings suggest that, whilst employing organisations might consider it appealing to screen for resilience among staff who wish to engage in crisis response or disaster relief work, this may well not be an effective way to protect the mental health of disaster relief teams. With few exceptions, the evidence base underlying the variables that might be included in such screening is weak. Given that risks also exist for screening, including the risk of inappropriately excluding skilled staff and of enhancing stigma or discrimination against those identified as high risk [86], employers may wish to exert caution when using screening tools until better quality evidence relating to their use become available. Our findings suggest that in some cases, careful scrutiny of individuals who appear vulnerable by appropriately skilled occupational health staff is warranted.

\section{Strengths and limitations}

The main strengths of this review are the detailed systematic search strategy, which identified multiple relevant studies, and the high quality of the studies included in the review.

As with all systematic reviews, however, there is a risk of publication bias and selective reporting. The risk of publication bias was heightened by our decision to only include studies published in peer-reviewed journals and published in English. The exclusion of foreign-language or grey literature reports may have altered our conclusions.

Synthesis was made challenging in our review by the vague terminology used in some papers, the use of multiple outcome measurement tools, and the range of techniques used to define an adverse outcome. In particular, the use of distress as the primary endpoint in many studies posed a difficulty. Distress, at least in the shortterm, is a normal response to a traumatic exposure and does not necessarily indicate the presence of any mental health disorder. On this basis, pre-employment screening for risk factors for distress may be uncalled for. On the other hand, distressed employees may require additional support from their colleagues and line manager, making identification of risk factors a worthwhile exercise. In this review, we elected to include studies relating to both distress and disorder. Although we include both types of outcome in our synthesis, our impression is that excluding distress would not have dramatically altered our conclusions. It would be helpful if future studies could look at the risk of experiencing longer term illness rather than short term distress.

The majority of the studies included in this review were cross-sectional, relying on retrospective recall of pre-employment and pre-deployment predictors thus leaving the studies vulnerable to recall bias. More prospective studies are required if further investigation into risk factors is desired.

The common use of self-report measures to assess post-deployment symptomatology also leaves studies open to criticism, as this is not equivalent to diagnosis by a qualified clinician. However, a recent meta-analysis of PTSD prevalence amongst emergency service workers found that both self-report and diagnostic interviews provided similar results [91].

\section{Conclusion}

Identifying members of staff who are likely to experience distress or disorder following disaster relief work is a laudable aim. However, in practice, the current evidence base does not support the use of pre-employment and pre-deployment screening as a method of protecting disaster workers' mental health. Our results do support disaster response organisations ensuring that volunteers are as well prepared and supported as professional disaster works. Additionally, people with a prior history of mental illness, or who have recently experienced significant life stressors, should be scrutinised by occupational health staff to ensure that any associated risks are properly managed. However, our results suggest that until better evidence becomes available, there is no role for the use of pre-employment and pre-deployment mental health screening for this population.

\section{Supplementary information}

Supplementary information accompanies this paper at https://doi.org/10. 1186/s12888-020-02593-1.

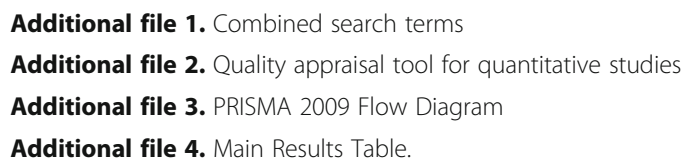

\section{Abbreviations}

PTSD: Post-traumatic stress disorder; USA: United States of America; EO: Elena Opie; SKB: Samantha Brooks; NG: Neil Greenberg; GJR: G. James Rubin

\section{Acknowledgements \\ Not applicable.}

\section{Authors' contributions}

EO carried out the data searches, screening, data extraction and data analysis, and drafted the manuscript. SKB contributed to the development of the search strategy. SKB, NG and GJR participated in the design and coordination of the study and made suggestions after reading the initial draft of the manuscript. All authors read and approved the final manuscript. 


\section{Funding}

The UK Public Health Rapid Support Team, funded by UK aid from the Department of Health and Social Care, is a partnership between Public Health England (PHE) and the London School of Hygiene \& Tropical Medicine (LSHTM). University of Oxford and King's College London are academic partners. The views expressed are those of the author(s) and not necessarily those of their employers, the NHS, the NIHR or the Department of Health and Social Care.

\section{Availability of data and materials}

The datasets supporting the conclusions of this article are included within the article and its additional files.

\section{Ethics approval and consent to participate}

Not applicable.

\section{Consent for publication}

Not applicable.

\section{Competing interests}

NG runs a psychological consultancy, which works with organisations that employ disaster workers. The other authors declare that they have no competing interests.

Received: 10 May 2019 Accepted: 12 April 2020

Published online: 11 May 2020

\section{References}

1. Bakhshi S, Lynn-Nicholson R, Jones B, Amlôt R, Greenberg N, Rubin GJ. Responding to a radiological crisis: Experiences of British foreign office staff in Japan after the Fukushima nuclear meltdown. Disaster Med Public Health Preparedness. 2014;8(5):397-403.

2. Clukey L. Transformative experiences for hurricanes Katrina and Rita disaster volunteers. Disasters. 2010;34(3):644-56.

3. Perrin MA, DiGrande L, Wheeler K, Thorpe L, Farfel M, Brackbill R. Differences in PTSD prevalence and associated risk factors among world trade center disaster relief and recovery workers. Am J Psychiatry. 2007;164(9):1385-94.

4. Soliman HH, Lingle, S. E., \& Raymond, A. Perceptions of indigenous workers following participation in a disaster relief project. Community Mental Health Journal. 1998(34(6)):557-68.

5. Thoresen S, Tonnessen A, Lindgaard CV, Andreassen AL, Weisaeth L. Stressful but rewarding: Norwegian personnel mobilised for the 2004 tsunami disaster. Disasters. 2009:33(3):353-68.

6. Yokoyama Y, Hirano K, Sato M, Abe A, Uebayashi M, Kishi E, et al. Activities and health status of dispatched public health nurses after the great East Japan earthquake. Public Health Nurs. 2014;31(6):537-44.

7. Chang C-M, Lee L-C, Connor KM, Davidson JR, Jeffries K, Lai T-J. Posttraumatic distress and coping strategies among rescue workers after an earthquake. J Nerv Ment Dis. 2003;191(6):391-8.

8. Shah SA, Garland E, Katz C. Secondary traumatic stress: prevalence in humanitarian aid workers in India. Traumatology. 2007;13(1):59-70.

9. Hagh-Shenas H, Goodarzi MA, Dehbozorgi G, Farashbandi H. Psychological consequences of the bam earthquake on professional and nonprofessional helpers. J Traumatic Stress. 2005;18(5):477-83.

10. Soldatos CR, Paparrigopoulos TJ, Pappa DA, Christodoulou GN. Early posttraumatic stress disorder in relation to acute stress reaction: an ICD-10 study among help seekers following an earthquake. Psychiatry Res. 2006;143(2-3): 245-53.

11. Bryant RA, Guthrie RM. Maladaptive self-appraisals before trauma exposure predict posttraumatic stress disorder. J Consult Clin Psychol. 2007;75(5):812

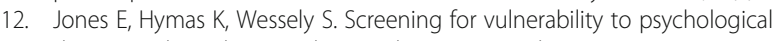
distress in the military: an historical survey. J Med Screen. 2003;10:40-6.

13. Rona R, Hyams C, Wessely S. Screening for psychological illness in military personnel. J Am Med Assoc. 2005;293:1257-60.

14. Rona R, Jones M, Hooper R, Hull L, Browne T, Horn O, Murphy D, Hotopf M, Wessely S. Mental health screening in armed forces before the Iraq war and prevention of subsequent psychological morbidity: a follow-up study. $\mathrm{Br}$ Med J. 2006:333:991-9.

15. Marshall RE, Milligan-Saville JS, Mitchell PB, Bryant RA, Harvey SB. a systematic review of the usefulness of pre-employment and pre-duty screening in predicting mental health outcomes amongst emergency workers. Psychiatry Res. 2017;253:129-37.

16. Moher D, Liberati A, Tetzlaff J, Altman DG. Preferred reporting items for systematic reviews and meta-analyses: the PRISMA statement. Ann Intern Med. 2009;151(4):264-9.

17. Reuters T EndNote X7. Thomson Reuters: Philadelphia, PA, USA. 2013(16).

18. Brooks SKDR, Sage CAM, Amlôt R, Greenberg N, Rubin J. Risk and resilience factors affecting the psychological wellbeing of individuals deployed in humanitarian relief roles after a disaster. J Ment Health. 2015;24(6):385-413.

19. Drummond MJT. Guidelines for authors and peer reviewers of economic submissions to the BMJ. The BMJ economic evaluation working party. $\mathrm{Br}$ Med J. 1996;313:275-83.

20. Quality Assessment Tool for Quantitative Studies. Effective Public Healthcare Panacea Project. 2020. Available from: https://www.ephpp.ca/qualityassessment-tool-for-quantitative-studies/. Cited 2 May 2020.

21. Musa SA, Hamid AA. Psychological problems among aid workers operating in Darfur. Soc Behav Personal Int J. 2008:36(3):407-16.

22. Stellman JM, Smith RP, Katz CL, Sharma V, Charney DS, Herbert R, et al. Enduring mental health morbidity and social function impairment in world trade center rescue, recovery, and cleanup workers: the psychological dimension of an environmental health disaster. Environ Health Perspect. 2008;116(9):1248.

23. Bowler RM, Harris M, Li J, Gocheva V, Stellman SD, Wilson K, et al. Longitudinal mental health impact among police responders to the 9/11 terrorist attack. Am J Ind Med. 2012;55(4):297-312.

24. Sim K, Chua HC. The psychological impact of SARS: a matter of heart and mind. Can Med Assoc J. 2004;170(5):811-2.

25. Bowler RM, Han H, Gocheva V, Nakagawa S, Alper H, DiGrande L, et al. Gender differences in probable posttraumatic stress disorder among police responders to the 2001 world trade center terrorist attack. Am J Ind Med. 2010;53(12):1186-96.

26. Chang C-M, Lee L-C, Connor KM, Davidson JR, Lai T-J. Modification effects of coping on post-traumatic morbidity among earthquake rescuers. Psychiatry Res. 2008:158(2):164-71.

27. Jayasinghe N, Giosan C, Evans S, Spielman L, Difede J. Anger and posttraumatic stress disorder in disaster relief workers exposed to the 9/11/ 01 world trade center disaster: one-year follow-up study. J Nerv Ment Dis. 2008;196(11):844.

28. Pietrzak RH, Schechter CB, Bromet EJ, Katz CL, Reissman DB, Ozbay F, et al. The burden of full and subsyndromal posttraumatic stress disorder among police involved in the world trade center rescue and recovery effort. J Psychiatr Res. 2012;46(7):835-42.

29. Feder A, Mota N, Salim R, Rodriguez J, Singh R, Schaffer J, et al. Risk, coping and PTSD symptom trajectories in world trade center responders. J Psychiatr Res. 2016;82:68-79.

30. Pietrzak R, Feder A, Singh R, Schechter C, Bromet EJ, Katz C, et al. Trajectories of PTSD risk and resilience in world trade center responders: an 8-year prospective cohort study. Psychol Med. 2014;44(1):205-19.

31. Epstein RS, Fullerton CS, Ursano RJ. Posttraumatic stress disorder following an air disaster: a prospective study. Am J Psychiatr. 1998;155(7):934-8.

32. McFarlane AC. The aetiology of post-traumatic morbidity: predisposing precipitating and perpetuating factors. Br J Psychiatry. 1989;154(2):221-8.

33. Wu P, Fang Y, Guan Z, Fan B, Kong J, Yao Z, et al. The psychological impact of the SARS epidemic on hospital employees in China: exposure, risk perception, and altruistic acceptance of risk. Can J Psychiatry. 2009:54(5): 302-11

34. Zhen Y. Huang Z-q, Jin J, Deng X-y, Zhang L-p, Wang J-g. posttraumatic stress disorder of red cross nurses in the aftermath of the 2008 Wenchuan China earthquake. Arch Psychiatr Nurs. 2012:26(1):63-70.

35. Cukor J, Wyka K, Jayasinghe N, Weathers F, Giosan C, Leck P, et al. Prevalence and predictors of posttraumatic stress symptoms in utility workers deployed to the world trade center following the attacks of September 11, 2001. Depression and anxiety. 2011;28(3):210-7.

36. Adams RE, Figley CR, Boscarino JA. The compassion fatigue scale: its use with social workers following urban disaster. Res Soc Work Pract. 2008;18(3): 238-50.

37. Armagan E, Engindeniz Z, Devay AO, Erdur B, Ozcakir A. Frequency of posttraumatic stress disorder among relief force workers after the tsunami in Asia: do rescuers become victims? Prehospital Disaster Med. 2006;21(3):168-72. 
38. Ben-Ezra M, Essar N, Saar R. Post-traumatic reactions among rescue personnel 96 hours after the Hilton Hotel bombing in Sinai: the effect of previous exposure. Stress Health. 2005;21(4):269-72.

39. Ben-Ezra M, Palgi $Y$, Essar $N$, Sofer $H$, Haber $Y$. Acute stress symptoms, dissociation, and depression among rescue personnel 24 hours after the bet-Yehoshua train crash: the effects of exposure to dead bodies. Prehospital Disaster Med. 2008;23(5):461-5.

40. Creamer $T L$, Liddle BJ. Secondary traumatic stress among disaster mental health workers responding to the September 11 attacks. J Traumatic Stress. 2005;18(1):89-96

41. Dobashi K, Nagamine M, Shigemura J, Tsunoda T, Shimizu K, Yoshino A, et al. Psychological effects of disaster relief activities on Japan ground selfdefense force personnel following the 2011 great East Japan earthquake. Psychiatry. 2014;77(2):190-8.

42. Fullerton CS, Ursano RJ, Wang L. Acute stress disorder, posttraumatic stress disorder, and depression in disaster or rescue workers. Am J Psychiatr. 2004; 161(8):1370-6.

43. Grieger TA, Fullerton CS, Ursano RJ, Reeves JJ. Acute stress disorder, alcohol use, and perception of safety among hospital staff after the sniper attacks. Psychiatr Serv. 2003;54(10):1383-7.

44. Huang J, Liu Q, Li J, Li X, You J, Zhang L, et al. Post-traumatic stress disorder status in a rescue group after the Wenchuan earthquake relief. Neural Regen Res. 2013;8(20):1898.

45. McFarlane AC. The longitudinal course of posttraumatic morbidity: the range of outcomes and their predictors. J Nerv Ment Dis. 1988.

46. Misra M, Greenberg N, Hutchinson C, Brain A, Glozier N. Psychological impact upon London ambulance service of the 2005 bombings. Occup Med. 2009;59(6):428-33.

47. Razik S, Ehring T, Emmelkamp PM. Psychological consequences of terrorist attacks: prevalence and predictors of mental health problems in Pakistani emergency responders. Psychiatry Res. 2013;207(1-2):80-5.

48. Thormar SB, Gersons BP, Juen B, Djakababa MN, Karlsson T, Olff M. Organizational factors and mental health in community volunteers. The role of exposure, preparation, training, tasks assigned, and support. Anxiety Stress Coping. 2013;26(6):624-42.

49. Tosone C, McTighe JP, Bauwens J, Naturale A. Shared traumatic stress and the long-term impact of 9/11 on Manhattan clinicians. J Trauma Stress. 2011;24(5):546-52.

50. Tucker P, Pfefferbaum B, Doughty DE, Jordan FB, Jones DE, Nixon SJ. Body handlers after terrorism in Oklahoma City: predictors of posttraumatic stress and other symptoms. Am J Orthopsychiatry. 2002;72(4):469-75.

51. Ursano RJ, Fullerton CS, Kao T-C, Bhartiya V. Longitudinal assessment of posttraumatic stress disorder and depression after exposure to traumatic death. J Nervous Mental Dis. 1995.

52. van der Velden PG, Kleber RJ, Koenen KC. Smoking predicts posttraumatic stress symptoms among rescue workers: a prospective study of ambulance personnel involved in the Enschede fireworks disaster. Drug Alcohol Depend. 2008;94(1-3):267-71.

53. Wang H, Jin H, Nunnink SE, Guo W, Sun J, Shi J, et al. Identification of post traumatic stress disorder and risk factors in military first responders 6 months after wen Chuan earthquake in China. J Affect Disord. 2011;130(12):213-9.

54. Ehring T, Razik S, Emmelkamp PM. Prevalence and predictors of posttraumatic stress disorder, anxiety, depression, and burnout in Pakistani earthquake recovery workers. Psychiatry Res. 2011;185(1-2):161-6.

55. Maunder RG, Lancee WJ, Balderson KE, Bennett JP, Borgundvaag B, Evans S, et al. Long-term psychological and occupational effects of providing hospital healthcare during SARS outbreak. Emerg Infect Dis. 2006;12(12): 1924.

56. Ben-Ezra M, Essar N, Saar R. The association between peritraumatic dissociation and acute stress reactions among rescue personnel 36-48h after a gas pipe explosion in Tel-Aviv: a preliminary report. Stress and Health: Journal of the International Society for the Investigation of Stress. 2006;22(3):197-201.

57. Boscarino JA, Figley CR, Adams RE. Compassion fatigue following the September 11 terrorist attacks: a study of secondary trauma among New York City social workers. International journal of emergency mental health. 2004;6(2):57.

58. Lundin T, Bodegard M. The psychological impact of an earthquake on rescue workers: A follow-up study of the Swedish group of rescue workers in Armenia. 1993:1988.
59. Biggs QM, Fullerton CS, Reeves JJ, Grieger TA, Reissman D, Ursano RJ. Acute stress disorder, depression, and tobacco use in disaster workers following 9/ 11. Am J Orthopsychiatry. 2010;80(4):586-92.

60. Liao S-C, Lee M-B, Lee $Y$-J, Weng T, Shih F-Y, Ma MH. Association of psychological distress with psychological factors in rescue workers within two months after a major earthquake. J Formos Med Assoc. 2002;101(3): 169-76.

61. Hodgkinson PE, Shepherd MA. The impact of disaster support work. J Trauma Stress. 1994;7(4):587-600.

62. McFarlane AC. Life events and psychiatric disorder: the role of a natural disaster. Br J Psychiatry. 1987;151(3):362-7.

63. Spinhoven $P$, Verschuur M. Predictors of fatigue in rescue workers and residents in the aftermath of an aviation disaster: a longitudinal study. Psychosom Med. 2006;68(4):605-12.

64. Marmar CR. Characteristics of emergency services personnel related to peritraumatic dissociation during critical incident exposure. Am J Psychiatry. 1996;153(7):94.

65. Alvarez J, Hunt M. Risk and resilience in canine search and rescue handlers after 9/11. Journal of Traumatic Stress: Official Publication of The International Society for Traumatic Stress Studies. 2005;18(5):497-505.

66. Evans S, Patt I, Giosan C, Spielman L, Difede J. Disability and posttraumatic stress disorder in disaster relief workers responding to September 11, 2001 world trade center disaster. J Clin Psychol. 2009:65(7):684-94.

67. North CS, Tivis L, McMillen JC, Pfefferbaum B, Spitznagel EL, Cox J, et al. Psychiatric disorders in rescue workers after the Oklahoma City bombing. Am J Psychiatr. 2002;159(5):857-9.

68. Alexander DA, Wells A. Reactions of police officers to body-handling after a major disaster a before-and-after comparison. Br J Psychiatry. 1991;159(4): 547-55.

69. Dougall AL, Herberman HB, Delahanty DL, Inslicht SS, Baum A. Similarity of prior trauma exposure as a determinant of chronic stress responding to an airline disaster. J Consult Clin Psychol. 2000;68(2):290.

70. Dyregrov A, Kristoffersen Jl, Gjestad R. Voluntary and professional disasterworkers: similarities and differences in reactions. J Trauma Stress. 1996;9(3): 541-55

71. Ersland $S$, Weisæth $L$, Sund $A$. The stress upon rescures involved in an oil rig disaster."Alexander L. Kielland" 1980. Acta Psychiatr Scand. 1989;80:38-49.

72. Hytten K, Hasle A. Fire fighters: a study of stress and coping. Acta Psychiatr Scand. 1989;80:50-5.

73. Weiss DS, Marmar CR, Metzler TJ, Ronfeldt HM. Predicting symptomatic distress in emergency services personnel. J Consult Clin Psychol. 1995;63(3):361.

74. van der Velden PG, van Loon P, Benight CC, Eckhardt T. Mental health problems among search and rescue workers deployed in the Haïti earthquake 2010: a pre-post comparison. Psychiatry Res. 2012;198(1):100-5.

75. Marmar CR, Weiss DS, Metzler TJ, Delucchi KL, Best SR, Wentworth KA. Longitudinal course and predictors of continuing distress following critical incident exposure in emergency services personnel. J Nerv Ment Dis. 1999; 187(1):15-22.

76. Bartone PT, Ursano RJ, Wright KM, Ingraham LH. The impact of a military air disaster on the health of assistance workers. J Nerv Ment Dis. 1989;177(6): 317-28.

77. Lu Y-C, Shu B-C, Chang Y-Y. The mental health of hospital workers dealing with severe acute respiratory syndrome. Psychother Psychosom. 2006;75(6): 370-5.

78. Maunder R. The experience of the 2003 SARS outbreak as a traumatic stress among frontline healthcare workers in Toronto: lessons learned. Philosophical Transactions of the Royal Society of London B: Biological Sciences. 2004;359(1447):1117-25.

79. Hagh-Shenas H, Goodarzi MA, Dehbozorgi G, Farashbandi H. Psychological consequences of the bam earthquake on professional and nonprofessional helpers. Journal of Traumatic Stress: Official Publication of The International Society for Traumatic Stress Studies. 2005;18(5):477-83.

80. Brown J, Mulhern G, Joseph S. Incident-related stressors, locus of control, coping, and psychological distress among firefighters in Northern Ireland. J Trauma Stress. 2002:15(2):161-8.

81. Cieslak R, Shoji K, Luszczynska A, Taylor S, Rogala A, Benight CC. Secondary trauma self-efficacy: concept and its measurement. Psychol Assess. 2013, 25(3):917.

82. Christiansen DM, Hansen M. Accounting for sex differences in PTSD: a multivariable mediation model. Eur J Psychotraumatol. 2015;6(1):26068. 
83. Roberts AL, Gilman SE, Breslau J, Breslau N, Koenen KC. Race/ethnic differences in exposure to traumatic events, development of post-traumatic stress disorder, and treatment-seeking for post-traumatic stress disorder in the United States. Psychol Med. 2011:41(1):71-83.

84. Carlson EB, Palmieri PA, Field NP, Dalenberg CJ, Macia KS, Spain DA. Contributions of risk and protective factors to prediction of psychological symptoms after traumatic experiences. Compr Psychiatry. 2016;69:106-15.

85. National Institute for Health and Care Excellence (2015) Workplace health: management practices (NICE guideline NG13). Available at: https:/www.nice. org.uk/guidance/ng13 [.

86. Brooks SK, Dunn R, Sage CA, Amlôt R, Greenberg N, Rubin GJ. Risk and resilience factors affecting the psychological wellbeing of individuals deployed in humanitarian relief roles after a disaster. J Ment Health. 2015: 24(6):385-413.

87. Sippel LM, Pietrzak RH, Charney DS, Mayes LC, Southwick SM. How does social support enhance resilience in the trauma-exposed individual? Ecology and Society. 2015;20(4).

88. Bonabi H, Müller M, Ajdacic-Gross V, Eisele J, Rodgers S, Seifritz E, Rössler W, Rüsch N. Mental health literacy, attitudes to help seeking, and perceived need as predictors of mental health service use: a longitudinal study. J Nerv Ment Dis. 2016 Apr 1;204(4):321-4.

89. Guilaran J, de Terte I, Kaniasty K, Stephens C. Psychological outcomes in disaster responders: a systematic review and meta-analysis on the effect of social support. International Journal of Disaster Risk Science. 2018 Sep 1;9(3): 344-58.

90. Berger W, Coutinho ESF, Figueira I, Marques-Portella C, Luz MP, Neylan TC et al. Rescuers at risk: a systematic review and meta-regression analysis of the worldwide current prevalence and correlates of PTSD in rescue workers. Soc Psychiatry Psychiatr Epidemiol. 2012;47(6):1001-11.

91. Pachman J. Evidence base for pre-employment medical screening. Bull World Health Organ. 2009;87:529-34.

\section{Publisher's Note}

Springer Nature remains neutral with regard to jurisdictional claims in published maps and institutional affiliations.

Ready to submit your research? Choose BMC and benefit from:

- fast, convenient online submission

- thorough peer review by experienced researchers in your field

- rapid publication on acceptance

- support for research data, including large and complex data types

- gold Open Access which fosters wider collaboration and increased citations

- maximum visibility for your research: over $100 \mathrm{M}$ website views per year

At $\mathrm{BMC}$, research is always in progress.

Learn more biomedcentral.com/submissions 